7-1-2017

\title{
Biochemical Effects of Exercise on a Fasciocutaneous Flap in a Rat Model.
}

\author{
Edita Aksamitiene \\ Thomas Jefferson University \\ Adam L. Baker \\ Thomas Jefferson University \\ Sudeep Roy \\ Thomas Jefferson University \\ Salini Hota \\ Thomas Jefferson University
}

Follow this and additional works at: https://jdc.jefferson.edu/otofp

Li-Hul Zhang

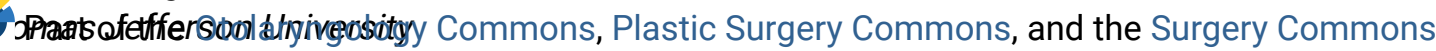

Let us know how access to this document benefits you

See next page for additional authors

\section{Recommended Citation}

Aksamitiene, Edita; Baker, Adam L.; Roy, Sudeep; Hota, Salini; Zhang, Li-Hui; Rodin, Julianna; Hobelmann, Kealan; Hoek, Jan B.; and Pribitkin, Edmund A., "Biochemical Effects of Exercise on a Fasciocutaneous Flap in a Rat Model." (2017). Department of Otolaryngology - Head and Neck Surgery Faculty Papers. Paper 37.

https://jdc.jefferson.edu/otofp/37

This Article is brought to you for free and open access by the Jefferson Digital Commons. The Jefferson Digital Commons is a service of Thomas Jefferson University's Center for Teaching and Learning (CTL). The Commons is a showcase for Jefferson books and journals, peer-reviewed scholarly publications, unique historical collections from the University archives, and teaching tools. The Jefferson Digital Commons allows researchers and interested readers anywhere in the world to learn about and keep up to date with Jefferson scholarship. This article has been accepted for inclusion in Department of Otolaryngology - Head and Neck Surgery Faculty Papers by an authorized administrator of the Jefferson Digital Commons. For more information, please contact: JeffersonDigitalCommons@jefferson.edu. 


\section{Authors}

Edita Aksamitiene, Adam L. Baker, Sudeep Roy, Salini Hota, Li-Hui Zhang, Julianna Rodin, Kealan Hobelmann, Jan B. Hoek, and Edmund A. Pribitkin 
JAMA Facial Plastic Surgery | Original Investigation

\section{Biochemical Effects of Exercise on a Fasciocutaneous Flap in a Rat Model}

Edita Aksamitiene, PhD; Adam L. Baker, MD; Sudeep Roy, MD; Salini Hota, BS; Li-Hui Zhang, BS; Julianna Rodin, BA; Kealan Hobelmann, BS; Jan B. Hoek, PhD; Edmund A. Pribitkin, MD, MBA

IMPORTANCE An overwhelming amount of data suggest that cardiovascular exercise has a positive effect on the mind and body, although the precise mechanism is not always clear.

OBJECTIVE To assess the clinical and biochemical effects of voluntary cardiovascular exercise on pedicled flaps in a rodent model.

DESIGN, SETTING, AND PARTICIPANTS Eighteen adult Sprague-Dawley male rats were randomized into a resting animal group (RAG) $(n=9)$ and an exercise animal group (EAG) $(n=9)$ for 14 days (July 23, 2013, through July 30, 2013). A pedicled transposition flap was performed on the ventral surface of the rat, and biopsy specimens were taken from the proximal, middle, and distal portions on postoperative days $0,2,5$, and 9 . Flap survival was analyzed planimetrically, and biopsy specimens were analyzed by hematoxylin-eosin-stained microscopy and immunoblotting. The housing, exercise, surgery, and analysis of the rats were conducted at a single basic science research laboratory at the tertiary care center campus of Thomas Jefferson University in Philadelphia, Pennsylvania.

EXPOSURES The rats were caged for 14 days or housed in a cage connected to an exercise wheel and pedometer.

MAIN OUTCOMES AND MEASURES Study measures were gross and micrographic necrosis and expression of proteins within cell survival and apoptosis pathways.

RESULTS A total of 18 rats were studied, 9 in the RAG and 9 in the EAG. the mean (SEM) amount of necrosis in flaps was $41.3 \%(3 \%)$ in the RAG rats and $10.5 \%(3.5 \%)$ in the EAG rats $(P<.001)$. Immunoblotting revealed increased Caspase-9 activity resulting in

poly-(adenosine diphosphate-ribose) polymerase 1 cleavage in the RAG vs the EAG, as well as lower phosphorylated protein kinase B (also known as Akt), signal transducer and activator of transcription 3, and total B-cell leukemia/lymphoma 2 protein levels. Throughout the postoperative period, the cumulative vascular endothelial growth factor A levels of the EAG flaps were significantly higher than those of the RAG flaps ( 2.30 vs 1.25 fold induction [FI], $P=.002$ ), with differences of $2.76 \mathrm{vs} 1.54 \mathrm{Fl}$ in the proximal segment, $2.40 \mathrm{vs} 1.20 \mathrm{Fl}$ in the middle segment, and 1.90 vs $0.79 \mathrm{Fl}$ in the distal segment. A similar response was noted when comparing phosphorylated Akt, with cumulative mean (SEM) p-Akt expression levels of 0.62 (0.04) for RAG and 1.98 (0.09) for EAG ( $P=.002$ between the 2 groups).

CONCLUSIONS AND RELEVANCE Voluntary preoperative exercise improves survival in pedicled fasciocutaneous flaps; the EAG rats had less necrosis, decreased apoptotic markers, and increased amounts of vascular endothelial growth factor $A$ and prosurvival proteins. These results have implications to increase flap survival in other mammal populations, such as humans.

LEVEL OF EVIDENCE 3.
Supplemental content

Author Affiliations: Department of Otolaryngology-Head and Neck Surgery, Sidney Kimmel Medical College, Thomas Jefferson University, Philadelphia, Pennsylvania (Aksamitiene, Baker, Roy, Hota, Zhang, Rodin, Hobelmann, Pribitkin); Department of Pathology, Anatomy, and Cell Biology, Sidney Kimmel Medical College, Thomas Jefferson University, Philadelphia, Pennsylvania (Hoek).

Corresponding Author: Edmund A. Pribitkin, MD, MBA, Department of Otolaryngology-Head and Neck Surgery, Thomas Jefferson University Hospital, 925 Chestnut St, Sixth Floor, Philadelphia, PA 19107 (edmund.pribitkin@jefferson.edu). 
A n overwhelming amount of data suggest that cardiovascular exercise has a positive effect on the mind and body, ${ }^{1}$ although the precise mechanism is not always clear. Several studies ${ }^{2-4}$ have found that cardiovascular exercise hastens wound healing in not only the rodent population but also humans. Indeed, the effect on humans varies from biochemical and immunologic measures to emotional and cognitive effects. ${ }^{5}$ Emery et $\mathrm{al}^{2}$ found a significant acceleration in wound healing in an elderly population after a 3-month regimen of exercise vs a sedentary control group. These studies reveal the need for further research into the mechanisms through which these effects on wound healing are achieved.

Cells adapt and maintain oxygen homeostasis while tissue heals by altering the expression of up to 2000 hypoxiasensitive genes. ${ }^{6,7}$ Such genes include the vascular endothelial growth factor (VEGF) ${ }^{8}$ a potent endothelial cell-specific survival factor, and heat shock proteins. ${ }^{9}$ In response to VEGF binding, the VEGF receptor (VEGFR) undergoes autophosphorylation and transmits metabolic signals via phosphatidylinositol 3-kinase (PI3K)/protein kinase B (also known as Akt), a signal transducer and activator of transcription 3 (STAT3), Src family kinases (SFKs), nitric oxide synthase (NOS), phospholipase C- $\gamma$ (PLC- $\gamma$ ), mitogen-activated protein kinase (MAPK), and other signaling pathways. ${ }^{10-13}$ Activation of these signaling cascades inhibits cell death, promotes cell cycle progression with DNA synthesis, and stimulates cell motility. These cascades also induce angiogenesis, increase vascular permeability, and enhance autocrine VEGF production. ${ }^{14-16}$ Similarly, induction of various heat shock proteins promotes hypoxic tolerance and increases cell survival via modulation of the PI3K/Akt signaling ${ }^{17}$ and other cell death-related cellular mechanisms. ${ }^{18}$ Thus, creating an oxygen gradient elicits potent molecular responses that promote cell adaptation to injury and expedite wound repair. ${ }^{19}$

While under acute hypoxia, wounds transition through the 4 stages of wound healing (hemostasis, inflammation, proliferation, and maturation) in a consecutive manner. Repeated ischemia-reperfusion injury or chronic hypoxia results in wounds that are often trapped in the self-sustaining inflammatory stage and fail to progress. ${ }^{20}$ This setting of prolonged ischemia in surgical flap reconstruction will lead to necrosis and partial or full flap loss. In this article, we attempt to explain some of these positive effects of preoperative cardiovascular exercise using a local pedicled flap rat model to identify the biochemical changes that occur within the dynamic process of wound healing.

\section{Methods}

\section{Animals}

After 14 days (July 23, 2013, through July 30, 2013) of acclimatization, eighteen 6-month-old male Sprague-Dawley rats were split into 2 main experimental groups, both of which were provided water and identical food types ad libitum. The 9 rats in the exercising animal group (EAG) (initial mean weight, 500.4 g) were housed individually in cages with access to a running wheel and distance counter for 2 weeks before surgery (August 6, 2013,

\section{Key Points}

Question What are the clinical and biochemical effects of exercise on the survival of pedicled fasciocutaneous flaps in a rat model?

Findings In this experimental study comparing pedicled flap survival in 9 sedentary vs 9 voluntarily exercised adult male rats, exercise significantly decreased the amount of flap necrosis. Exercise dose-dependent flap survival correlated with statistically significant increased vascular endothelial growth factor levels and associated biochemical protein expression.

Meaning Voluntary presurgical exercise increases pedicled fasciocutaneous flap survival in rats.

through August 20,2013), whereas the 9 rats in the resting animal group (RAG) (initial mean weight, $507.6 \mathrm{~g}$ ) were housed in regular cages. The mean weights before surgery were $557.5 \mathrm{~g}$ in the RAG rats and $522.0 \mathrm{~g}$ in the EAG rats. The EAG was further divided into 2 subgroups: the longer distance exercise (LDE) rats, who traveled approximately $37 \mathrm{~km}(\mathrm{n}=5)$, and the shorter distance exercise (SDE) rats, who traveled approximately $15.5 \mathrm{~km}$ $(n=4)$. The rats did not exercise postoperatively. The animal exercise and surgery protocol was approved by the Institutional Animal Care and Use Committee, Thomas Jefferson University, Philadelphia, Pennsylvania.

\section{Single-Pedicled Transposition Flap Surgery}

As previously described by Luginbuhl et al, ${ }^{21}$ a vertically oriented, rectangular, $3 \times 8$-cm fasciocutaneous flap based off the inferior epigastric artery was designed, raised, transposed, and sutured to a defect site. The epigastric artery was identified and preserved in each case. The defect was an $8 \times 3$-cm fullthickness segment that was removed, snap frozen, and later referred to as the baseline level. Then $8 \times 8$-mm punch biopsy specimens were taken on the second and fifth postoperative days (PODs), and the whole flap was harvested on POD 9 (end point), photographed (for gross estimation of percentage of necrosis by planimetry), and separated into proximal, middle, and distal thirds (segments). Segments were snap frozen and stored at $-80^{\circ} \mathrm{C}$ before analysis. The principles of the animal model and the timeline of the experimental procedures are shown in Figure 1.

\section{Sample Analysis by Immunoblotting}

Tissue samples from each experimental group were individually crushed into fine powder under liquid nitrogen and then homogenized in $1 \mathrm{~mL}$ of ice-cold tissue lysis buffer $(50 \mathrm{mM}$ HEPES [4-(2-hydroxyethyl)-1-piperazineethanesulfonic acid], $\mathrm{pH} 7.4,150 \mathrm{mM}$ sodium chloride, $1 \mathrm{mM}$ ethyleneglycoltetraacetic acid, $1 \%$ Triton X-100, 0.5\% sodium deoxycholate, 0.1\% sodium dodecyl sulfate, and $10 \%$ glycerol supplemented with protease and phosphatase inhibitor cocktails [A. G. Scientific Inc]) per $100 \mathrm{mg}$ of tissue using a 5-mL Potter-Elvehjem glass homogenizer and grinder. The lysates were then prepared and separated by electrophoresis and analyzed by conventional immunoblotting or multistrip Western blotting. These methods are described in full detail in the eMethods and eFigure 1 in the Supplement. 


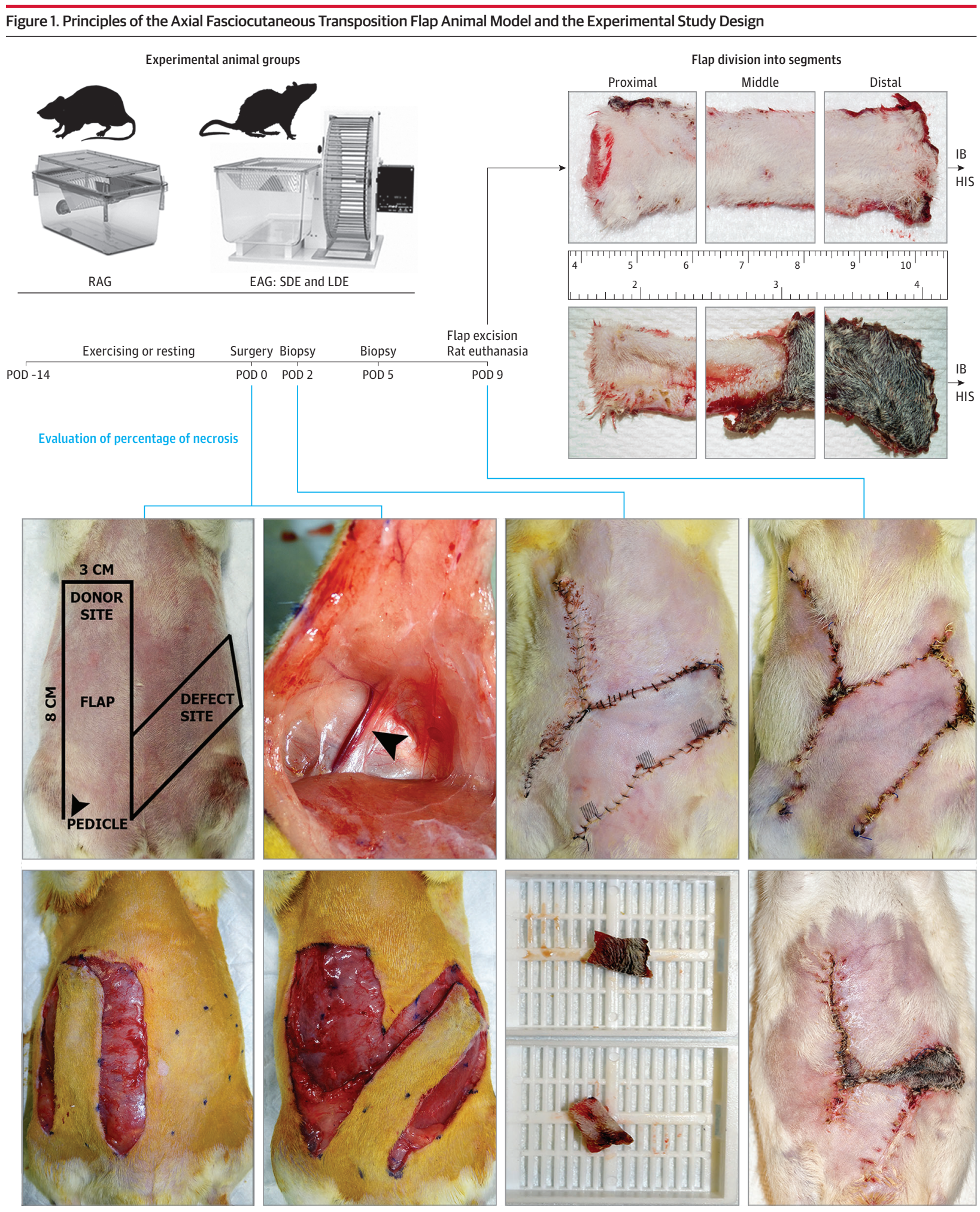

Locations for biopsy excision are identified by gray squares at the bottom of each flap segment (seen on POD 2 image). Arrowheads indicate the pedicle and its vessel (superficial epigastric inferior artery and vein) bundle. Upper ruler shows centimeters, lower ruler shows inches. EAG indicates exercise animal group; HIS, histologic analysis; IB, immunoblotting; LDE, longer distance exercise; POD, postoperative day; RAG, resting animal group; and SDE, shorter distance exercise. 
Histologic and Planimetric Analysis

Each strip that was taken from the middle section of each flap segment after excision and division was placed into separate histologic cassettes that were incubated in $10 \%$ buffered formalin (StatLab Medical Products) for 48 hours, washed with phosphatebuffered saline, and stored in $70 \%$ ethanol solution. Each specimen was embedded in paraffin and sectioned longitudinally. Three 4- $\mu \mathrm{m}$ tissue sections were placed onto each slide, which were then stained with hematoxylin-eosin (H\&E). Microphotographs of stained tissues were taken with a polarized microscope (BX-51-P; Olympus Corp). A series of digital photographs were presented in random fashion to a qualified, masked observer in the Department of Pathology, Anatomy, and Cell Biology, Sidney Kimmel Medical College, Thomas Jefferson University, Philadelphia, Pennsylvania, to assess gross necrosis. Gross necrosis was defined as nonviable, motteled, blackened, or escharlike skin. Flap survival was calculated by taking the total, necrotic, and viable flap areas in the photoimage of an intact excised flap delineated with the aid of the precision scale and ruler tool in Adobe Photoshop software (Adobe Systems Inc). The percentage of flap survival was calculated as the necrotic flap area minus the total flap area divide by the total flap area (in square millimeters) multiplied by 100 .

Statistical Analysis and Data Normalization

One-way analysis of variance with the Holm-Šídák test was used for statistical comparative analysis of planimetry data among all groups, and a 2-tailed $t$ test was used to compare the data obtained by immunoblotting analysis. The differences between signal intensity values of each animal group were considered to be statistically significant at 2 -sided $P<.05$ molecular protein weightlap segment. The signal intensities (in arbitrary units) of the phosphorylated protein of interest were first normalized to the signal intensities of the corresponding total protein, then normalized to a reference protein control (averaged $\beta$-actin and growth factor receptor-bound protein 2 signal intensities) and subsequently divided by the likewise normalized value of a baseline sample obtained at POD 0. Obtained signal intensities of nonphosphorylated proteins of interest were normalized to a reference protein control and then divided by the normalized value of baseline sample obtained at POD 0. Normalized values were expressed as the fold induction (FI) over the baseline level. Kinetic curves were plotted based on fold changes in GraphPad Prism statistical software (GraphPad Software Inc).

\section{Results}

A total of 18 rats were studied, 9 in the RAG and 9 in the EAG. The EAG was further subdivided into 4 SDE rats and 5 LDE rats. We estimated the effects of cardiovascular physical activity on axial-based fasciocutaneous flap survival by calculating the percentage of gross necrosis in intact flaps that were excised from each rat on POD 9. Figure 2A shows that rats that voluntarily exercised for 14 days on a running wheel before surgery had a 3.93fold difference in flap survival rate. The mean (SEM) percentage of necrosis of flaps was $41.3 \%$ (3\%) in the RAG rats and $10.5 \%$ (3.5\%) in the EAG rats $(P<.001)$. Comparing flap survival be- tween the RAG and the 2 subgroups of the EAG revealed a mean (SEM) percentage of necrosis of $19.3 \%(3.9 \%)$ in the SDE rats and $3.4 \%(2.7 \%)$ in the LDE rats. Accordingly, a significant difference was found between flap survival in the RAG vs EAG $(P<.001)$, RAG vs LDE $(P<.001)$, RAG vs SDE $(P=.002)$, and SDE vs LDE rats $(P=.045$ at a power of 0.970). Compared with the sedentary rats, the rats that exercised, and in particular those that ran longer distances, had a smaller number of partially failed flaps and more modest inflammatory cell infiltrate in their proximal and middle segments as verified by histologic analysis (Figure 2B). The H\&E-stained specimens in the most distal segments of the RAG exhibited pathologic signs of necrosis, such as acellular eosinophilic infiltrate without nuclei.

Figure 3A shows a representative flap from the RAG rats, which underwent biochemical analysis of cell survival-associated proteins. The flap noted had pronounced signs of necrosis in the middle and distal segments (Figure 3A). Compared with their respective basal levels, the active (phosphorylated) forms of Akt and STAT3 were decreased in the distal and most necrotic segment of the flap. This area coincided with the lowest relative abundance of antiapoptotic B-cell leukemia/lymphoma 2 (Bcl-2) protein expression (Figure $3 \mathrm{~A}$ ) and the highest abundance of active proapoptotic cysteine protease caspase-9. In addition, a decreasing amount of full-length poly-(adenosine diphosphate-ribose) polymerase (PARP) 1 and a concomitant increase of the cleaved form, which is a well-recognized marker for apoptotic cells, ${ }^{22,23}$ were found in the middle and distal segments. Pooled samples of both the RAG (titled without exercise) and EAG (titled with exercise) were analyzed for cell death-associated protein markers (Figure 3B). Presurgical exercise markedly suppressed enzymatic activity of caspase-9, whereas Bcl-2 levels had unaltered expression (Figure 3B). PARP-1 cleavage in distal flap segments of the EAG compared with the RAG had a similar suppression to that of caspase-9 (Figure 3B).

Figure 4 is a plot of prosurvival serine/threonine kinase Akt activation. Values were expressed as FI of dually phosphorylated Akt (p-Akt) over baseline levels detected by immunoblotting of the pooled RAG or EAG lysates. These values were similar to those obtained from individual samples. At POD 2, which is early in the inflammatory phase of wound healing, the activation levels of p-Akt decreased in all flap segments of the RAG and EAG below baseline, except in the proximal segments of exercised rats, which had a modest increase. By POD 9, p-Akt of the RAG proximal segments only slightly surpass basal levels in the RAG (approximately 1.2-fold). The p-Akt levels in the EAG sample pool exhibited a prominent increase of 3-fold in the proximal segment, 2.3-fold in the middle segment, and 2-fold in the distal segment over basal levels. On POD 9, the differences in Akt induction between the RAG and EAG were 2.7 -fold $(1.25$ vs $3.35 \mathrm{FI}, P<.001)$ in the proximal segment, 2.85 -fold ( 2.49 vs $0.87 \mathrm{FI}, P<.001)$ in the middle segment, and 3.2-fold ( 2 vs $0.63 \mathrm{FI}, P<.001$ ) in the distal segment. The cumulative mean (SEM) p-Akt expression levels across all flap portions throughout the entire experiment were 0.62 (0.04) for RAG and 1.98 (0.09) for EAG, which is an approximately 3.2 -fold difference $(P=.002$, unpaired parametric 2 -tailed $t$ test with unequal variance). Figure 4 illustrates VEGFA levels detected by immunoblotting of pooled homog- 
Figure 2. Effects of Voluntary Cardiovascular Exercise on Pedicled Fasciocutaneous Flap Survival

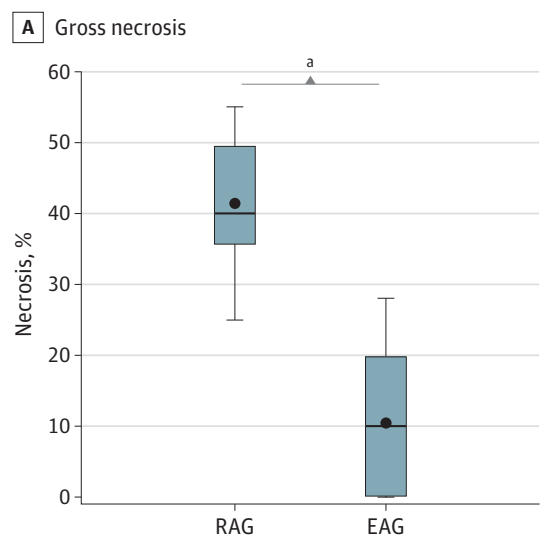

B Histologic specimens
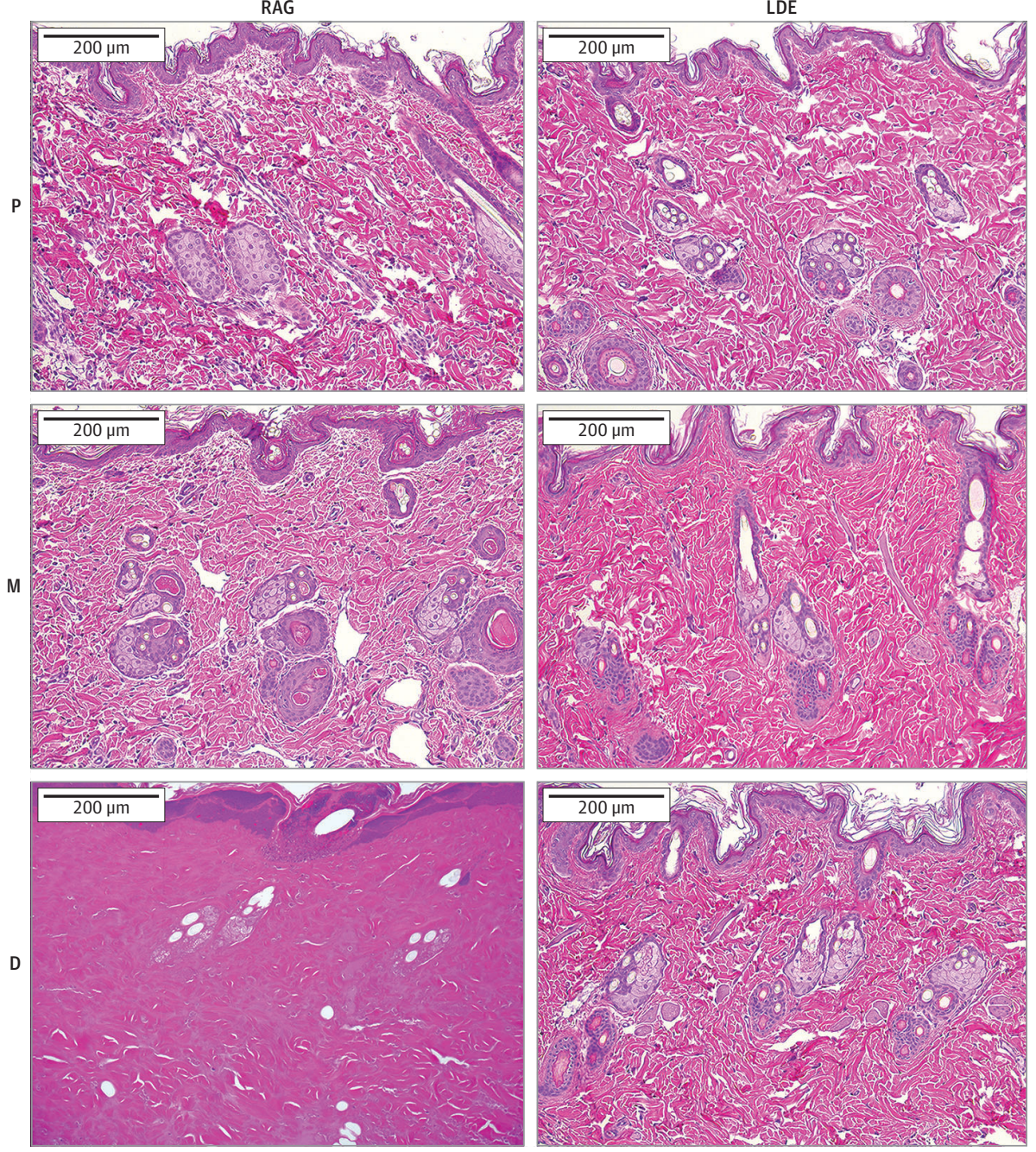

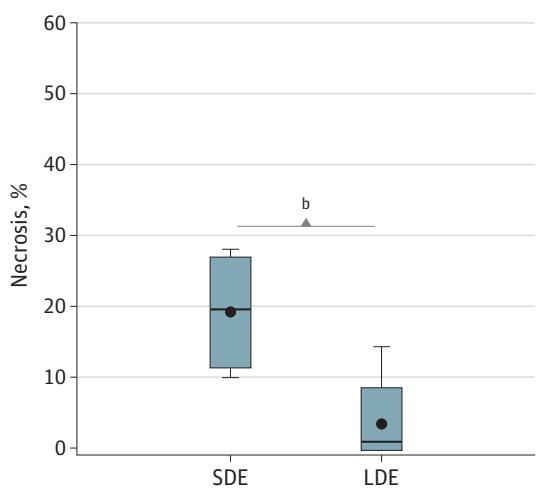

IDE

A, Comparison of gross necrosis percentage between the resting animal group (RAG) $(n=9)$ and the exercise animal group (EAG) $(n=9)$ and between the shorter distance exercise (SDE) $(n=4)$ and longer distance exercise (LDE) groups and EAG subgroups. Each box plot shows the median (horizontal line), mean (black circle), maximum (upper whisker), and minimum (bottom whisker) values. B, Representative hematoxylin-eosin slides of proximal $(P)$, middle (M), and distal (D) flap segments of the RAG and LDE group. a $P<.001$.

${ }^{\mathrm{b}} P<.05$. enates of the biopsy specimens of the flaps harvested from the RAG or EAG on different PODs. Rats that exercised had slightly higher baseline levels of VEGF than resting rats. The most robust and sustained VEGF level increase over baseline was observed in the proximal segments of the EAG (2-fold for POD 2, 2.8-fold on POD 5, and 3.3-fold for POD 9). The distal segments of the RAG had the weakest response to the surgery (0.5fold on POD 2, 0.8-fold on POD 5, and 1-fold on POD 9). On POD 9, the differences in the fold increase for VEGF over basal levels were 1.8-fold for proximal (3.30 vs 1.80, $P<.001$ ), 2 -fold 
Figure 3. Immunoblotting and Cell Death-Associated Protein Markers

A RAG flap appearance and IB results for cell survival proteins Proximal

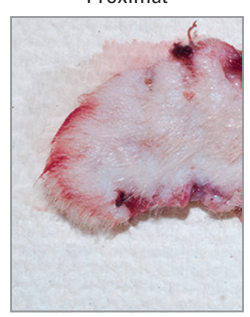
Middle
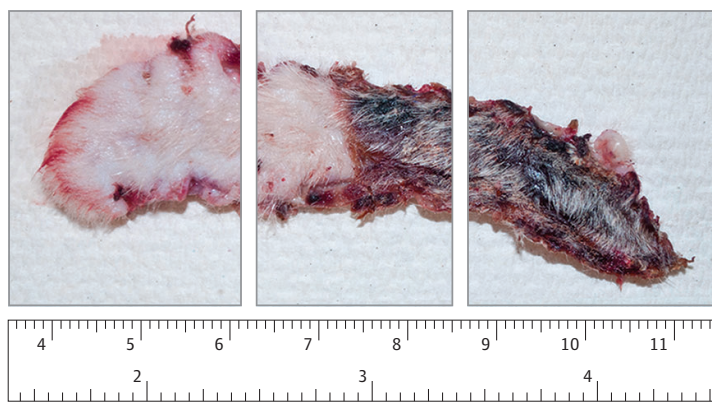

Distal

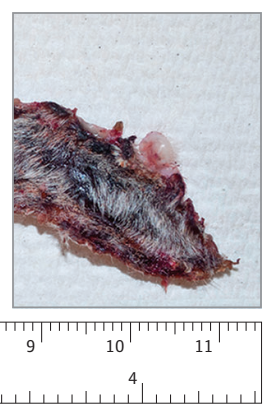

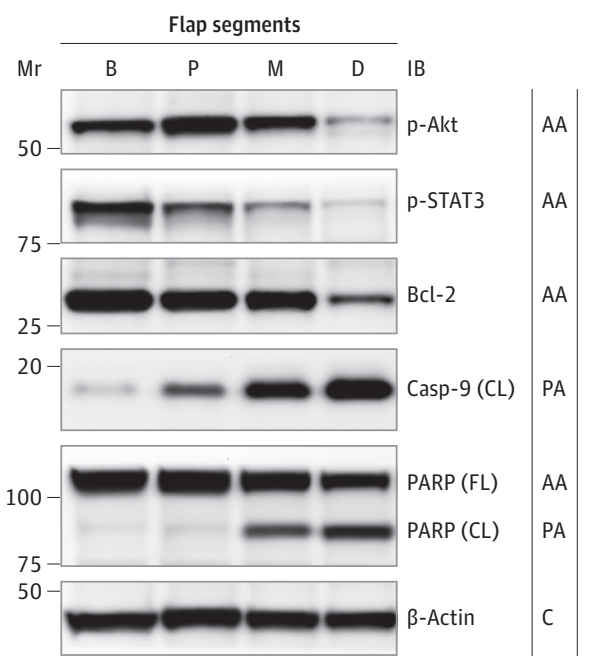

B RAG vs EAG cell death-associated protein markers and IB results

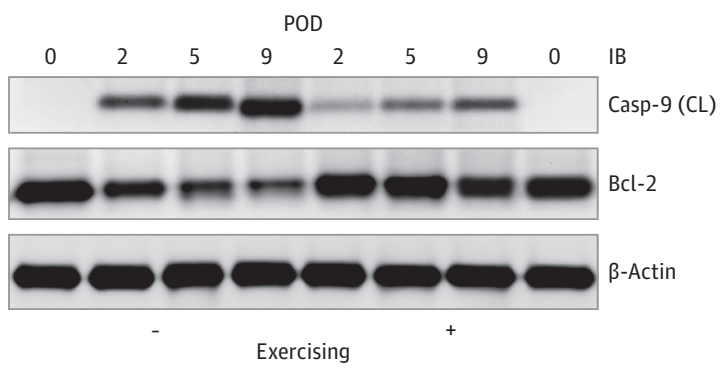

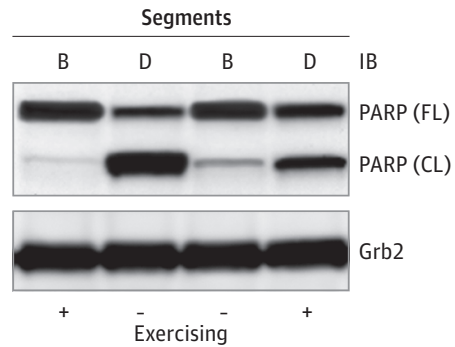

A, Gross appearance and immunoblotting (IB) of cell survival proteins of a resting animal group (RAG) flap on postoperative day (POD) 9. Left, The proximal $(P)$, middle $(M)$, and distal $(D)$ segments of the flap. The upper ruler shows centimeters, and the lower ruler shows inches. Right, The proapototic (PA), antiapototic (AA), and loading control $(C)$ proteins detected by IB in the baseline (B), P, M, and D segments. B, Cell death-associated protein markers in pooled samples of the RAG (minus sign) and exercise animal group (EAG) (plus sign) rats. Bcl-9 indicates B-cell leukemia/lymphoma 2; Casp-9, Caspase-9; $\mathrm{CL}$, cleaved; FL, full length; Grb2, growth factor receptor-bound protein 2; Mr, molecular protein weight; p-Akt, phosphorylated protein kinase B; PARP, poly-(adenosine diphosphate-ribose) polymerase 1; and p-STAT3, phosphorylated signal transducer and activator of transcription 3. for middle (2.84 vs 1.40, $P<.001)$, and 2.5 -fold for distal $(2.54$ vs 1.02, $P=.001$ ) segments. Throughout the postoperative period, the cumulative VEGF levels of the EAG flaps were significantly higher than those of the RAG flaps, with a difference of 1.8-fold (2.30 vs $1.25 \mathrm{FI}, P=.002$, 2-tailed unpaired $t$ test with unequal variance) between the 2 groups and differences of 1.8 -fold for the proximal segment ( $2.76 \mathrm{vs} 1.54 \mathrm{FI}$ ), 2 -fold for the middle segment (2.40 vs $1.20 \mathrm{FI}$ ), and 2.4-fold for the distal segment (1.90 vs $0.79 \mathrm{FI})$.

\section{Discussion}

Delaying flaps to improve vascularization before transposition has long been clinically found to improve flap survival. Similarly, increased exercise-dependent flap vascularization before surgery could be responsible for improved flap survival, but the mechanism for such an effect remains undescribed. Vascular endothelial growth factor is a critical survival factor for vascular endothelium, which promotes angiogenesis, interacts with a multitude of other growth fac- tors, and is thus essential for flap survival. ${ }^{14,15,23,24}$ The kinetics of VEGF expression during postoperative pedicled flap healing have not been previously reported to our knowledge. Our study found that VEGF expression is induced at the earliest stages of wound healing. The most robust response was found in the proximal and most healthy flap segment of exercising rats followed by the middle and distal segments of this group. The VEGF response of the distal segment of the EAG group was greater than all segments of the flap in the RAG (Figure 4). The observation that VEGF levels increased during each POD of the study suggests that VEGF synthesis is steadily increasing during the endothelial cell mitogenesis phase and is then maintained during the vascular remodeling phase (additional time points are required to determine whether VEGF is at peak). Not only did we note an increase in VEGF levels in all segments of the flap in the EAG, but we also found more activation of VEGFR (eFigure 2 in the Supplement) and less necrosis, both grossly and histologically (Figure 2). Furthermore, there appears to be a dose-dependent effect of the amount of exercise performed noted on flap necrosis (Figure 2A) and released VEGF levels (eFigure 3 in the Supplement). We observed a small but sig- 
Figure 4. Effects of Exercise on Flap Phosphorylated Protein Kinase B (p-Akt) Activation and Vascular Endothelial Growth Factor A (VEGFA) Expression

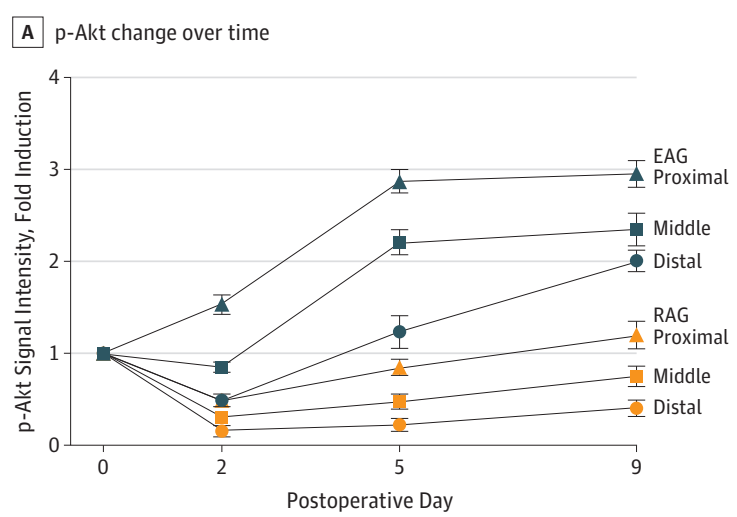

These graphs illustrate the relative fold increase over the baseline level of activated (Ser-473 or Thr-308) p-Akt and VEGFA as detected by multistrip Western blotting in pooled protein samples of the proximal, middle, or distal segments obtained at postoperative days 2, 5, and 9 or from excised defect during surgery (postoperative day 0 ) in the resting animal group (RAG) and exercise animal group (EAG). A, p-Akt signal intensity values detected were first

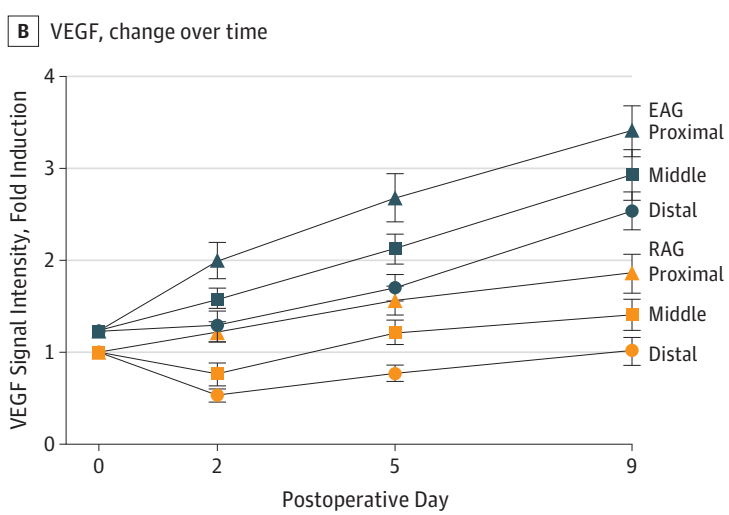

normalized to p-Akt, growth factor receptor-bound protein 2 (Grb2), and $\beta$-actin mean signal intensity values, averaged and then plotted as fold induction (FI) in reference to basal p-Akt levels. B, Densitometric VEGFA signal intensity values were normalized to Grb2 and $\beta$-actin signal intensity mean values and then plotted as FI in reference to baseline VEGF levels. Data are expressed as mean (SD) $(68 \% \mathrm{CI})$ from a technical triplicate. nificant mean (SEM) difference in flap necrosis between the $\operatorname{SDE}(19.3 \%$ [3.9\%]) and LDE (3.4\% [2.7\%]) groups.

Increased levels of p-Akt, a potent activator of the cell survival pathway, were also noted (Figure 4). The increase in Akt activation promotes cell cycle progression and DNA synthesis and inhibits cell death. ${ }^{10,25,26}$ Figure 3 A shows the link between the gross postsurgical clinical appearance of the flap with biochemical results via immunoblotting. The most necrotic portions of the flap have high amounts of proapoptotic proteins, such as caspase- 9 and cleaved PARP, and decreased amounts of antiapoptotic proteins, such as Bcl-2 and pSTAT3. As expected, p-Akt levels were decreased in the necrotic portion of the flap (Figure $3 \mathrm{~A}$ ). When we pooled the samples of the RAG and EAG to compare the proteins in the apoptosis pathway (Figure 3B), our data indicated that preoperative exercise decreased the proteolytic activity of caspase- 9 and maintained steady levels of Bcl-2-a downstream target of both Akt and STAT3 that is known to prevent the effect of caspase-9. ${ }^{26,27}$ This is juxtaposed to the RAG, which again exhibited increased proapoptotic proteins and decreased Bcl-2 (Figure 3B).

Although the exact mechanism for this apparent induction noted by exercising rats (Figure 4 ) is not entirely clear, such activation has been previously described in response to exercise-induced stress ${ }^{17,28}$ and likely reflects increased VEGFR activation in response to exercise (eFigure 2 in the Supplement). Perhaps cells exposed to higher levels of stress during more strenuous exercise can escalate VEGF synthesis de novo, prolong VEGF messenger RNA half-life, or protect existing VEGF molecules from degradation and thus sustain VEGFR signaling (eFigure 3 in the Supplement). Such upregulation of VEGF may depend on successful concomitant activation of STAT3 (eFigure 3 in the Supplement), which aside from mediating prosurvival and anti-inflammatory and proinflammatory responses ${ }^{29,30}$ is known to bind to a VEGF gene pro- moter and induce angiogenesis. ${ }^{31,32}$ Moreover, intense exercise markedly augmented phosphorylated endothelial nitric oxide synthase levels (eFigure 3 in the Supplement). Activation of this enzyme in endothelial cells by Akt-dependent phosphorylation downstream of activated VEGFR produces secondary messenger nitric oxide, which improves vessel vasodilation. ${ }^{33-35}$ Therefore, the flaps after physical activity are expected to be more vascular and better perfused, but additional studies using histologic staining and fluorescence or combined power Doppler and photoacoustic imaging are needed to estimate these values in vital portions of flap tissue. In addition, whether the results in this experiment are localized to the abdomen or reflect systemic effects of exercise must also be clarified.

These clinical and biochemical results suggest that preoperative exercise can lead to improved flap survival or, at the very least, that sedentary behavior is conducive for necrosis and ultimately partial flap failure. Although our data may attempt to explain the mechanism of previous reports ${ }^{1-4}$ of the benefits of exercise in wound healing, we have only begun to scratch the surface. Our results suggest that preoperative cardiovascular exercise may improve flap outcomes in patients undergoing reconstructive surgery. However, additional studies must be completed to further explain the many biochemical pathways that link the two.

\section{Limitations}

Limitations of this study included the small sample size and the need to pool certain specimens to perform biochemical analyses. For example, the tendency for rats to exercise could not be controlled. Some rats exercised more than others; the unknown factors that led the rats to exercise more could have resulted in better flap survival and more robust VEGF expression rather than the exercise itself. Pooling of samples was required to compare proteins within the apoptosis pathway, and 
such pooling may have underestimated (skewing because of a low outlier) or overestimated (skewing because of a high outlier) the exercise-associated decrease in proapoptotic protein expression. Moreover, although flap survival represented a clinically relevant end point, the study was unable to specifically correlate in vivo flap vascularity with VEGF expression or the mitigation of proapoptotic pathways.

\section{Conclusions}

Voluntary preoperative exercise improves survival in pedicled fasciocutaneous flaps in a rodent model. The exercise group had less gross necrosis, decreased apoptotic markers, and increased amounts of VEGF and cell survival proteins.

\section{ARTICLE INFORMATION}

Accepted for Publication: November 30, 2016.

Published Online: March 9, 2017

doi:10.1001/jamafacial.2016.2162

Author Contributions: Drs Aksamitiene and Baker contributed equally to this work. Drs Aksamitiene and Pribitkin had full access to all of the data in the study and take responsibility for the integrity of the data and the accuracy of the data analysis. Study concept and design: Aksamitiene, Roy, Hota, Zhang, Pribitkin.

Acquisition, analysis, or interpretation of data: All authors.

Drafting of the manuscript: Baker, Aksamitiene, Hota, Zhang, Rodin.

Critical revision of the manuscript for important intellectual content: Baker, Aksamitiene, Roy, Hota, Hobelmann, Hoek, Pribitkin.

Statistical analysis: Aksamitiene, Hota.

Obtained funding: Pribitkin.

Administrative, technical, or material support Baker, Hota, Rodin, Hobelmann, Hoek, Pribitkin. Study supervision: Baker, Roy, Pribitkin.

Conflict of Interest Disclosures: None reported.

Funding/Support: This study was supported by grant R01 AA018873 from the National Institutes of Health (Dr Hoek) and grant T32 AA007463 from the National Institute of Alcohol Abuse and Alcoholism (Dr Aksamitiene).

Role of the Funder/Sponsor: The funding sources had no role in the design and conduct of the study; collection, management, analysis, and interpretation of the data; preparation, review, or approval of the manuscript; and the decision to submit the manuscript for publication.

\section{REFERENCES}

1. Pate RR, Pratt M, Blair SN, et al. Physical activity and public health: a recommendation from the Centers for Disease Control and Prevention and the American College of Sports Medicine. JAMA. 1995; 273(5):402-407.

2. Emery CF, Kiecolt-Glaser JK, Glaser R, Malarkey WB, Frid DJ. Exercise accelerates wound healing among healthy older adults: a preliminary investigation. J Gerontol A Biol Sci Med Sci. 2005;60 (11):1432-1436.

3. Keylock KT, Vieira VJ, Wallig MA, DiPietro LA, Schrementi M, Woods JA. Exercise accelerates cutaneous wound healing and decreases wound inflammation in aged mice. Am J Physiol Regul Integr Comp Physiol. 2008;294(1):R179-R184.

4. Pence BD, Woods JA. Exercise, obesity, and cutaneous wound healing: evidence from rodent and human studies. Adv Wound Care (New Rochelle). 2014;3(1):71-79.

5. Hillman CH, Erickson KI, Kramer AF. Be smart, exercise your heart: exercise effects on brain and cognition. Nat Rev Neurosci. 2008;9(1):58-65.
6. Mense SM, Sengupta A, Zhou M, et al. Gene expression profiling reveals the profound upregulation of hypoxia-responsive genes in primary human astrocytes. Physiol Genomics. 2006;25(3):435-449

7. Semenza GL. Hypoxia-inducible factor 1: oxygen homeostasis and disease pathophysiology. Trends Mol Med. 2001;7(8):345-350.

8. Liu Y, Cox SR, Morita T, Kourembanas S. Hypoxia regulates vascular endothelial growth factor gene expression in endothelial cells: identification of a $5^{\prime}$ enhancer. Circ Res. 1995;77(3):638-643.

9. Baird NA, Turnbull DW, Johnson EA. Induction of the heat shock pathway during hypoxia requires regulation of heat shock factor by hypoxia-inducible factor-1. J Biol Chem. 2006;281 (50):38675-38681

10. Abid MR, Guo S, Minami T, et al. Vascular endothelial growth factor activates $\mathrm{PI} 3 \mathrm{~K} / \mathrm{Akt} /$ forkhead signaling in endothelial cells. Arterioscler Thromb Vasc Biol. 2004;24(2):294-300.

11. Chen SH, Murphy DA, Lassoued W, Thurston G, Feldman MD, Lee WM. Activated STAT3 is a mediator and biomarker of VEGF endothelial activation. Cancer Biol Ther. 2008;7(12):1994-2003.

12. Gee E, Milkiewicz M, Haas TL. p38 MAPK activity is stimulated by vascular endothelial growth factor receptor 2 activation and is essential for shear stress-induced angiogenesis. J Cell Physiol. 2010;222(1):120-126.

13. Koch S, Claesson-Welsh L. Signal transduction by vascular endothelial growth factor receptors. Cold Spring Harb Perspect Med. 2012;2(7):a006502.

14. Hoeben A, Landuyt $B$, Highley MS, Wildiers $H_{\text {, }}$ Van Oosterom AT, De Bruijn EA. Vascular endothelial growth factor and angiogenesis. Pharmacol Rev. 2004;56(4):549-580.

15. Kliche S, Waltenberger J. VEGF receptor signaling and endothelial function. IUBMB Life. 2001:52(1-2):61-66.

16. Olsson AK, Dimberg A, Kreuger J, Claesson-Welsh L. VEGF receptor signalling-in control of vascular function. Nat Rev Mol Cell Biol. 2006;7(5):359-371

17. Shiota M, Kusakabe H, Izumi Y, et al. Heat shock cognate protein 70 is essential for Akt signaling in endothelial function. Arterioscler Thromb Vasc Biol. 2010;30(3):491-497.

18. Nylandsted J, Gyrd-Hansen M, Danielewicz A, et al. Heat shock protein 70 promotes cell survival by inhibiting lysosomal membrane permeabilization. J Exp Med. 2004;200(4):425-435.

19. Pugh CW, Ratcliffe PJ. Regulation of angiogenesis by hypoxia: role of the HIF system. Nat Med. 2003;9(6):677-684

20. Lin Q, Cong $X$, Yun Z. Differential hypoxic regulation of hypoxia-inducible factors $1 a$ and $2 a$. Mol Cancer Res. 2011;9(6):757-765.
21. Luginbuhl $A$, Modest $M$, Yan K, Curry J, Heffelfinger R. Novel irradiated axial rotational flap model in the rodent. JAMA Facial Plast Surg. 2013;15(5):344-348.

22. Fink SL, Cookson BT. Apoptosis, pyroptosis, and necrosis: mechanistic description of dead and dying eukaryotic cells. Infect Immun. 2005;73(4):1907-1916.

23. Kano MR, Morishita Y, Iwata C, et al. VEGF-A and FGF-2 synergistically promote neoangiogenesis through enhancement of endogenous PDGF-B-PDGFRbeta signaling. J Cell Sci. 2005;118(pt 16):3759-3768

24. Salgado R, Benoy I, Bogers J, et al. Platelets and vascular endothelial growth factor (VEGF): a morphological and functional study. Angiogenesis. 2001;4(1):37-43.

25. Gao Y, Moten A, Lin HK. Akt: a new activation mechanism. Cell Res. 2014;24(7):785-786.

26. Pugazhenthi S, Nesterova A, Sable C, et al. Akt/protein kinase $\mathrm{B}$ up-regulates $\mathrm{BCl}-2$ expression through CAMP-response element-binding protein. J Biol Chem. 2000;275(15):10761-10766.

27. Sepúlveda P, Encabo A, Carbonell-Uberos $F$ Miñana MD. BCL-2 expression is mainly regulated by JAK/STAT3 pathway in human CD34+ hematopoietic cells. Cell Death Differ. 2007:14(2):378-380.

28. Dimmeler S, Assmus B, Hermann C, Haendeler J, Zeiher AM. Fluid shear stress stimulates phosphorylation of Akt in human endothelial cells: involvement in suppression of apoptosis. Circ Res. 1998;83(3):334-341. 29. El Kasmi KC, Holst J, Coffre M, et al. General nature of the STAT3-activated anti-inflammatory response. J Immunol. 2006;177(11):7880-7888.

30. Hirano T, Ishihara $K$, Hibi M. Roles of STAT3 in mediating the cell growth, differentiation and survival signals relayed through the IL- 6 family of cytokine receptors. Oncogene. 2000;19(21):2548-2556.

31. Chen Z, Han ZC. STAT3: a critical transcription activator in angiogenesis. Med Res Rev. 2008;28 (2):185-200

32. Niu G, Wright KL, Huang M, et al. ConstitutiveStat3 activity up-regulates VEGF expression and tumor angiogenesis. Oncogene. 2002;21(13):2000-2008. 33. Dimmeler $S$, Fleming I, Fisslthaler $B$, Hermann C, Busse R, Zeiher AM. Activation of nitric oxide synthase in endothelial cells by Akt-dependent phosphorylation. Nature. 1999;399(6736):601-605.

34. Maiorana A, O'Driscoll G, Taylor R, Green D. Exercise and the nitric oxide vasodilator system. Sports Med. 2003;33(14):1013-1035.

35. Blanes MG, Oubaha M, Rautureau Y, Gratton JP. Phosphorylation of tyrosine 801 of vascular endothelial growth factor receptor- 2 is necessary for Akt-dependent endothelial nitric-oxide synthase activation and nitric oxide release from endothelial cells. J Biol Chem. 2007;282(14):10660-10669.

36. Choi HJ, Lee JH, Park SY, Cho JH, Han JS. STAT3 is involved in phosphatidic acid-induced Bcl-2 expression in HeLa cells. Exp Mol Med. 2009;41(2):94-101. 